\title{
A Generalization of the Clark-Ocone Formula
}

\author{
Mahmmoud Salih*, Sulieman Jomah \\ School of Mathematics and Statistics, Northwest Normal University, Lanzhou, China \\ Email: *Mahmmoud.Salih@yahoo.com, suliemanjomah@hotmail.com
}

How to cite this paper: Salih, M. and Jomah, S. (2018) A Generalization of the Clark-Ocone Formula. Journal of Applied Mathematics and Physics, 6, 1443-1453. https://doi.org/10.4236/jamp.2018.67121

Received: June 5, 2018

Accepted: July 16, 2018

Published: July 19, 2018

Copyright (c) 2018 by authors and Scientific Research Publishing Inc. This work is licensed under the Creative Commons Attribution International License (CC BY 4.0).

http://creativecommons.org/licenses/by/4.0/

\section{Abstract}

In this paper, we use a white noise approach to Malliavin calculus to prove the generalization of the Clark-Ocone formula

$$
F(\omega)=E[F]+\int_{0}^{T} E\left[D_{t} F \mid \mathcal{F}_{t}\right] \diamond W(t) \mathrm{d} t,
$$

where $E[F]$ denotes the generalized expectation, $D_{t} F(\omega)=\frac{\mathrm{d} F}{\mathrm{~d} \omega}$ is the (generalized) Malliavin derivative, $\diamond$ is the Wick product and $W(t)$ is the 1-dimensional Gaussian white noise.

\section{Keywords}

White Noise, Malliavin Calculus, Wick Product, Brownian Motion

\section{Introduction}

In 1975, Hida introduced the theory of white noise with his lecture note on Brownian functionals [1]. After that H. Holden et al. [2] emphasized this theory with stochastic partial differential equations (SPDEs) driven by Brownian motion.

In 1984, Ocone proved the Clark-Ocone formula [3], to give an explicit representation to integral in Itô integral representation theorem in the context of analysis on the Wiener space $\Omega=C_{0}([0, T])$, the space of all real continuous functions on $[0, T]$ starting at 0 . He proved that

$$
F(\omega)=E[F]+\int_{0}^{T} E\left[D_{t} F \mid \mathcal{F}_{t}\right] \mathrm{d} B(t),
$$

where $D_{t}$ is the Malliavin derivative and $B(t)$ is the one dimensional Brownian motion on the Winer space. In [4] the authors proved the generalization of Clark-Ocone formula (see, e.g., [5] [6]). This theorem has many interesting application, for example, computing the replicating portfolio of call option in Black \& Scholes type market. They proved that 


$$
F(\omega)=E[F]+\int_{0}^{T} E\left[D_{t} F \mid \mathcal{F}_{t}\right] \diamond W(t) \mathrm{d} t
$$

where $E[F]$ denotes the generalized expectation, $D_{t} F(\omega)=\frac{\mathrm{d} F}{\mathrm{~d} \omega}$ is the (generalized) Malliavin derivative, $\diamond$ is the Wick product and $W(t)$ is the one dimensional Gaussian white noise. This formula holds for all $F \in \mathcal{G}^{*}$, where $\mathcal{G}^{*}$ is a space of stochastic distribution. In particular, if $F \in L^{2}(\mu)$ then equation (1.2) turns out to be

$$
F(\omega)=E[F]+\int_{0}^{T} E\left[D_{t} F \mid \mathcal{F}_{t}\right] \mathrm{d} B(t) .
$$

The purpose of this papper is to generalize the well known Clark-Ocone formula to generalized functions of white noise, i.e., to the space $\mathcal{G}^{-\beta}$. The generalization has the following form

$$
F(\omega)=E[F]+\int_{0}^{T} E\left[D_{t} F \mid \mathcal{F}_{t}\right] \diamond W(t) \mathrm{d} t,
$$

where $E[F]$ denotes the generalized expectation, $D_{t} F(\omega)=\frac{\mathrm{d} F}{\mathrm{~d} \omega}$ is the (generalized) Malliavin derivative, $\diamond$ is the Wick product, and $W(t)$ is the 1-dimensional Gaussian white noise.

The paper is organized as follows. In Section 2 and 3, we recall necessary definitions and results from white noise and prove a new results that we will need. Finally in Section 4, we generalize the Clark-Ocone formula, i.e., to the space $\mathcal{G}^{-\beta}$.

\section{White Noise}

In this section we recall necessary definitions and results from white noise. For more information about white noise analysis (see e.g, [7]-[14]).

Given $\Omega=S(\mathbb{R})$ be the space of tempered distribution on the set $\mathbb{R}$ of real number and let $\mu$ be the Gaussian white noise probability measure on $\Omega$ such that

$$
\int_{\Omega} \mathrm{e}^{i\langle\omega, \phi\rangle} \mathrm{d} \mu(\omega)=\mathrm{e}^{-\frac{1}{2} \mid \phi \|^{2}} .
$$

where $\langle\omega, \phi\rangle$ denotes the action of $\omega \in S^{\prime}(\mathbb{R})$ on $\phi$. It follows from (2.1) that

$$
E[\langle\cdot, \phi\rangle]=0, \quad E[\langle., \phi\rangle]^{2}=\|\phi\|^{2}, \phi \in S(\mathbb{R})
$$

where $E=E_{\mu}$ denotes the expectation with respect to $\mu$. This isometry allows us to define a Brownian motion $B(t)=B(t, \omega)$ as the continuous version of $\tilde{B}=\tilde{B}(t, \omega)=\left\langle\omega, \chi_{(0, t)}().\right\rangle$ where

$$
\chi_{[0, t]}(s)= \begin{cases}1 & \text { if } 1 \leq s \leq t \\ -1 & \text { if }-t \leq s \leq 0 \\ 0 & \text { otherwise }\end{cases}
$$

Then, $\langle\omega, \varphi\rangle=\int_{\mathbb{R}} \varphi(t) \mathrm{d} B(t)$ for all $\varphi \in L^{2}(\mathbb{R})$. Let $\mathcal{F}_{t}$ be the $\sigma$ algebra 
generated by $\{B(s, .)\}_{0 \leq s \leq t}$. If $f\left(t_{1}, t_{2}, \cdots, t_{n}\right) \in \hat{L}^{2}\left(\mathbb{R}^{n}\right)$, i.e., $f_{n}$ is symmetric and

$$
\left\|f_{n}\right\|_{L^{2}\left(\mathbb{R}^{n}\right)}=\int_{\mathbb{R}^{n}} f_{n}^{2}\left(t_{1}, \cdots, t_{n}\right) \mathrm{d} t_{1} \cdots \mathrm{d} t_{n}<\infty,
$$

then the iterated Itô integral is given by

$$
\int_{\mathbb{R}^{n}} f_{n} \mathrm{~d} B^{\otimes_{n}}:=n ! \int_{-\infty}^{\infty}\left(\int_{-\infty}^{t_{n}} \cdots\left(\int_{-\infty}^{t_{2}} f\left(t_{1}, \cdots, t_{n}\right) \mathrm{d} B\left(t_{1}\right)\right) \cdots\right) \mathrm{d} B\left(t_{n}\right) .
$$

In the following we let

$$
h_{n}(x)=(-1)^{n} \mathrm{e}^{\frac{x^{2}}{2}} \frac{\mathrm{d}^{n}}{\mathrm{~d} x^{n}}\left(\mathrm{e}^{-\frac{x^{2}}{2}}\right) ; n=0,1,2, \cdots
$$

be the Hermite polynomials and let $\left\{\xi_{n}\right\}_{n=1}^{\infty}$ be the basis of $L^{2}(\mathbb{R})$ consiting

$$
\xi_{n}(x)=\pi^{-\frac{1}{4}}((n-1) !)^{-\frac{1}{2}} \mathrm{e}^{-\frac{x^{2}}{2}} h_{n-1}(\sqrt{2} x), n=1,2, \cdots
$$

The set of multi-indices $\alpha=\left(\alpha_{1}, \alpha_{2}, \cdots, \alpha_{n}\right)$ of nonnegative integers is denoted by $\mathscr{T}=\left(\mathbb{N}_{0}^{\mathbb{N}}\right)_{\mathbb{C}}$. Where $\mathbb{N}=\{1,2, \cdots\}$ is the set of all natural number and $\mathbb{N}_{0}=\mathbb{N} \cup\{0\}$. If $z=\left(z_{1}, z_{2}, \cdots\right)$ is a sequence of number or function, we use the multi-induces notation

$$
z^{\alpha}=z_{1}^{\alpha_{1}} z_{2}^{\alpha_{2}} \cdots z_{n}^{\alpha_{n}} \text { if } \alpha=\left(\alpha_{1}, \cdots, \alpha_{n}\right) \in \mathscr{T}
$$

Theorem 2.1. ([15]) Let $\varphi_{1}, \varphi_{2}, \cdots, \varphi_{n}$ be are an orthonormal function in $L^{2}(\Omega)$. Then for all multi-indices $\alpha=\left(\alpha_{1}, \cdots, \alpha_{n}\right) \in \mathscr{T}$, we have

$$
\int_{\mathbb{R}^{|\alpha|}} \varphi^{\hat{\otimes} \alpha} \mathrm{d} B^{\otimes|\alpha|}(x)=h_{\alpha_{1}}\left(\left\langle\omega, \varphi_{1}\right\rangle\right) \cdots h_{\alpha_{n}}\left(\left\langle\omega, \varphi_{n}\right\rangle\right) .
$$

\section{Corollary 2.2 .}

$$
\left(H_{\alpha} \diamond H_{\beta}\right)=H_{\alpha+\beta}(\omega) ; \alpha, \beta \in \mathscr{T} .
$$

where $\diamond$ denote the Wick product, and extend linearly. Then if $f_{n} \in \hat{L}^{2}\left(\mathbb{R}^{n}\right), g_{n} \in \hat{L}^{2}\left(\mathbb{R}^{m}\right)$, we have

$$
\left(\sum_{n} \int_{\mathbb{R}^{n}} f_{n} \mathrm{~d} B^{\otimes n}\right) \diamond\left(\sum_{m} \int_{\mathbb{R}^{m}} g_{m} \mathrm{~d} B^{\otimes m}\right)=\sum_{m, n} \int_{\mathbb{R}^{m+n}} f_{n} \hat{\otimes} g_{m} \mathrm{~d} B^{\otimes(m+n)}
$$

Proof.

$$
\begin{aligned}
& \int_{\mathbb{R}^{|\alpha|}} \xi^{\hat{\otimes} \alpha} \mathrm{d} B^{\otimes|\alpha|} \diamond \int_{\mathbb{R}^{|\beta|}} \xi^{\hat{\otimes} \beta} \mathrm{d} B^{\otimes|\beta|} \\
& =H_{\alpha} \diamond H_{\beta}=H_{\alpha+\beta}=\int_{\mathbb{R}^{\mid \alpha+\beta} \mid} \xi^{\hat{\otimes}(\alpha+\beta)} \mathrm{d} B^{\otimes|\alpha+\beta|} \\
& =\int_{\mathbb{R}^{\mid \alpha+\beta} \xi^{\otimes \alpha}} \hat{\otimes} \xi^{\hat{\otimes} \beta} \mathrm{d} B^{\otimes|\alpha+\beta|} .
\end{aligned}
$$

\section{Stochastic Test Function and Stochastic Distribution (Konddratiev Spaces)}

1) Stochastic test function spaces

Suppose $k \in \mathbb{N}$, for $0 \leq \beta<1$, let $(S)_{\beta}$ consist of those

$$
f=\sum_{\alpha} c_{\alpha} H_{\alpha},
$$


such that

$$
\|f\|_{k, \beta}=\sum_{\alpha} c_{\alpha}^{2}(\alpha)^{1+\beta}(2 \mathbb{N})^{k \alpha}, \forall k \in \mathbb{N}
$$

where

$$
(2 \mathbb{N})^{k \alpha}=\prod_{i=1}^{m}(2 i)^{k \alpha_{i}}, \text { for } \alpha=\left(\alpha_{1}, \cdots, \alpha_{m}\right) .
$$

2) Stochastic distribution

For $0 \leq \beta<1$, let $(S)_{\beta}^{*}$ be the space of Kondratiev space of stochastic distribution, consist of all formal expansions

$$
F=\sum_{\alpha} b_{\alpha} H_{\alpha}
$$

such that

$$
\|F\|_{-q,-\beta}=\sum_{\alpha} b_{\alpha}^{2}(\alpha)^{1-\beta}(2 \mathbb{N})^{-q \alpha}, \text { for some } q \in \mathbb{N},
$$

where $(2 \mathbb{N})^{\alpha}$ is defined in (3.1).

Note that $(S)_{\beta}^{*}$ is the dual of $(S)_{\beta}$ and we can define the action of

$$
\begin{aligned}
& F=\sum_{\alpha} b_{\alpha} H_{\alpha} \in(S)_{\beta}^{*} \text { on } f=\sum_{\alpha} c_{\alpha} H_{\alpha} \in(S)_{\beta} \text { by } \\
& \langle F, f\rangle=\sum_{\alpha} \alpha !\left(b_{\alpha}, c_{\alpha}\right),
\end{aligned}
$$

where $\left(b_{\alpha}, c_{\alpha}\right)$ is the usual inner product in $\mathbb{R}$.

Definition 3.1. Let $F \in(S)_{\beta}^{*}$ be the random variable and let $\gamma \in L^{2}(\mathbb{R})$. Then we say that $F$ has directional derivative in the direction $\gamma$ if

$$
D_{\gamma} F(\omega):=\lim _{\epsilon \rightarrow 0} \frac{1}{\epsilon}(F(\omega+\epsilon \gamma)-F(\omega))
$$

if the limit exist in $F \in(S)_{\beta}^{*}$.

Definition 3.2. A function $\Phi: \mathbb{R} \rightarrow(S)_{\beta}^{*}$-integrable if

$$
\langle\Phi(.), \phi\rangle \in L^{1}(\mathbb{R}) \text {, for all } \phi \in(S)_{\beta} .
$$

Then the $(S)_{\beta}^{*}$-integrable of $\Phi(t)$, denoted by $\int_{\mathbb{R}} \Phi(t) \mathrm{d} t$, is the unique $(S)_{\beta}^{*}$ element such that

$$
\left\langle\int_{\mathbb{R}} \Phi(t) \mathrm{d} t, \phi\right\rangle=\int_{\mathbb{R}}\langle\Phi, \phi\rangle(t) \mathrm{d} t, \phi \in(S)_{\beta} .
$$

Definition 3.3. Consider $\varphi(t, \omega): \mathbb{R} \rightarrow(S)_{\beta}^{*}$ such that

$$
\varphi(t, \omega) \gamma(t) \text { is } \varphi(t, \omega) \text {-integrable }
$$

and

$$
D_{\gamma} F(\omega)=\int_{\mathbb{R}} \varphi(t, \omega) \gamma(t) \mathrm{d} t, \text { for all } \gamma \in L^{2}(\mathbb{R}),
$$

then we say that $F$ is (Hida) Malliavin differentiable and we put

$$
D_{t} F(\omega):=\frac{\mathrm{d} F}{\mathrm{~d} \omega}(t, \omega)=\varphi(t, \omega), t \in \mathbb{R} .
$$

$D_{t}$ is called the Hida-Malliavin derivative or stochastic gradient of $F$ at $t$. 
The set of all differentiable is denoted by $\mathbb{D}$.

Definition 3.4. Consider $F(\omega)=\sum_{\alpha} c_{\alpha} H_{\alpha}(\omega) \in(S)_{\beta}^{*}$. Then we define the stochastic derivative of $F$ at $t$ by

$$
\begin{aligned}
D_{t} F(\omega) & :=\frac{\mathrm{d} F}{\mathrm{~d} \omega}(t, \omega):=\sum_{\alpha} c_{\alpha} \sum_{i} \alpha_{i} H_{\alpha-\epsilon^{(i)}}(\omega) \cdot \xi_{i}(t) \\
& =\sum_{\gamma}\left(\sum_{i} c_{\gamma+\epsilon^{(i)}}\left(\gamma_{i}+1\right) \xi_{i}(t)\right) H_{\gamma}(\omega)
\end{aligned}
$$

\section{Lemma 3.5.}

1) Let $F \in(S)_{\beta}^{*}$. Then $D_{t} F \in(S)_{\beta}^{*}$ for a.a. $t \in \mathbb{R}$.

2) Suppose $F, F_{m} \in(S)_{\beta}^{*}$ for all $m \in \mathbb{N}$ and

$$
F_{m} \rightarrow F \operatorname{in}(S)_{\beta}^{*} .
$$

Then there exist a subsequence $\left\{F_{m_{k}}\right\}_{k=1}^{\infty}$ such that

$$
D_{t} F_{m_{k}} \rightarrow D_{t} F \text { in }(S)_{\beta}^{*} \text {, for } a . a t>0
$$

Proof. 1) Suppose $F(\omega)=\sum_{\alpha} c_{\alpha} H_{\alpha}(\omega) \in(S)_{\beta}^{*}$. Then

$$
\begin{aligned}
D_{t} F(\omega) & =\sum_{\alpha} c_{\alpha} \sum_{i} \alpha_{i} H_{\alpha-\epsilon^{(i)}}(\omega) \cdot \xi_{i}(t) \\
& =\sum_{\gamma}\left(\sum_{i} c_{\gamma+\epsilon^{(i)}}\left(\gamma_{i}+1\right) \xi_{i}(t)\right) H_{\gamma}(\omega) \\
& =\sum_{\gamma} g_{\gamma}(t) H_{\gamma}(\omega) .
\end{aligned}
$$

where $g_{\gamma}(t)=\sum_{i} c_{\gamma+\epsilon^{(i)}}\left(\gamma_{i}+1\right) \xi_{i}(t)$.

We want to prove that for some $q \in \mathbb{N}$,

$$
\left\|D_{t} F\right\|_{-\beta,-q-1}^{2}=\sum_{m}\left(\sum_{|\gamma|=m} g_{\gamma}^{2}(\gamma !)^{1-\beta}\right)(2 \mathbb{N})^{-\gamma(q+1)}<\infty \text { for } \text { a.a.t. }
$$

Note that

$$
\int_{\mathbb{R}} g_{\gamma}^{2}(t) \mathrm{d} t=\int_{\mathbb{R}}\left(\sum_{i} c_{\gamma+\epsilon^{(i)}}\left(\gamma_{i}+1\right) \xi_{i}(t)\right)^{2} \mathrm{~d} t=\sum c_{\gamma+\epsilon^{(i)}}^{2}\left(\gamma_{i}+1\right)^{2} .
$$

Moreover,

$$
(2 \mathbb{N})^{-\gamma q}<(2 \mathbb{N})^{-\gamma}=\prod_{i}(2 \cdot i)^{-\gamma_{i}} \leq \prod_{i} \mathrm{e}^{-\gamma_{i}(\log 2)}=\mathrm{e}^{-|\hat{\gamma}|}
$$

where $\tilde{\gamma}=(\log 2) \gamma_{i}$ for all $i \in I$. Hence,

$$
\begin{aligned}
& \int_{\mathbb{R}}\left\|D_{t} F\right\|_{-\beta,-q-1}^{2} \mathrm{~d} t \\
& =\sum_{\gamma}\left(c_{\gamma+\epsilon^{(i)}}^{2}\left(\gamma_{i}+1\right)^{2}(\gamma !)^{1-\beta}\right)(2 \mathbb{N})^{-\gamma(q+1)} \\
& =\sum_{\gamma, i}\left(\gamma_{i}+1\right)(\gamma !)^{-\beta}(2 \mathbb{N})^{-\gamma(q+1)} \sum_{\alpha,|\alpha|=|\gamma|+1} c_{\alpha}^{2} \alpha ! \\
& <\sum_{m} \sum_{|\tilde{y}|=m}(m+1) \mathrm{e}^{-m} \sum_{|\alpha|=(\log 2)^{-1} m+1} c_{\alpha}^{2}(\alpha !)(2 \mathbb{N})^{-\alpha q} .
\end{aligned}
$$


Using the fact that $(m+1) \mathrm{e}^{-m} \leq 1$ for all $m$, we get

$$
\int_{\mathbb{R}}\left\|D_{t} F\right\|_{-\beta,-q-1}^{2} \mathrm{~d} t<\sum_{m}\left(\sum_{|\alpha|=(\log 2)^{-1} m+1} c_{\alpha}^{2} \alpha !\right)(2 \mathbb{N})^{-\alpha q}<\|F\|_{-\beta,-q}<\infty .
$$

Therefore,

$$
D_{t} F \in(S)_{-\beta,-q-1} \text { for a.a. } t
$$

2) To prove this part, it suffices to prove that if $F_{m} \rightarrow 0$ in $(S)_{-\beta,-q}$, then there exist a subsequence $\left\{F_{m_{k}}\right\}_{k=1}^{\infty}$ such that $D_{t} F_{m} \rightarrow 0$ in $(S)_{\beta}^{*}$ as $k \rightarrow \infty$, for a.a. t. We have prove that

$$
\int_{\mathbb{R}}\left\|D_{t} F\right\|_{-\beta,-q-1}^{2} \mathrm{~d} t \leq\left\|F_{n}\right\|_{-\beta,-q}^{2} \rightarrow 0 .
$$

Therefore,

$$
\left\|D_{t} F_{n}\right\|_{-\beta,-q-1} \rightarrow 0 \text { in } L^{2}(\mathbb{R}) .
$$

So, there exists a subsequence $\left\{\left\|D_{t} F_{n}\right\|\right\}_{k \geq 1}$ such that $\left\|D_{t} F_{n_{k}}\right\|_{-\beta,-q-1} \rightarrow 0$ for a.a. $t$ as $k \rightarrow \infty$. This complete the proof.

Suppose $\xi_{1}, \xi_{2}, \cdots$ is the Hermite functions, and put

$$
X_{i}=X_{i}(\omega)=\left\langle\omega, \xi_{i}\right\rangle=\int_{\mathbb{R}} \xi_{i}(s) \mathrm{d} B(s) ; i=1,2, \cdots
$$

and

$$
X_{i}^{(t)}(\omega)=\int_{0}^{t} \xi_{i}(s) \mathrm{d} B(s) ; i=1,2, \cdots
$$

and

$$
X=\left(X_{1}, X_{2}, \cdots\right), X^{(t)}=\left(X_{1}^{(t)}, X_{2}^{(t)}, \cdots\right) .
$$

With this notation we have, $X^{\diamond \alpha}(\omega)=\left(X_{1}^{\diamond \alpha_{1}} \diamond \cdots \diamond X_{m}^{\diamond \alpha_{m}}\right)(\omega)=H_{\alpha}(\omega)$ for all multi indices $\alpha$ where $\alpha=\left(\alpha_{1}, \cdots, \alpha_{m}\right)$.

Definition 3.6. 1) Let $k \in \mathbb{N}, 0 \leq \beta<1$. We say that

$$
F=\sum_{n=0}^{\infty} I_{n}\left(f_{n}\right)
$$

belong to the space $\mathcal{G}_{k}^{\beta}$ if

$$
\|F\|_{\mathcal{G}_{k}^{\beta}}^{2}=\sum_{n=0}^{\infty} \mathrm{e}^{2 k n}(n !)^{1+\beta}\left\|f_{n}\right\|_{L^{2}\left(\mathbb{R}^{n}\right)}^{2}<\infty
$$

we define

$$
\mathcal{G}^{\beta}=\bigcap_{k \in \mathbb{N}} \mathcal{G}_{k}^{\beta},
$$

and equip $\mathcal{G}^{\beta}$ with the projective topology.

2) We say that

$$
G=\sum_{n=0}^{\infty} I_{n}\left(g_{n}\right)
$$

belong to the space $\mathcal{G}_{-q}^{-\beta}$ if 


$$
\|G\|_{\mathcal{G}_{-q}^{-\beta}}^{2}=\sum_{n=0}^{\infty} \mathrm{e}^{-2 q n}(n !)^{1-\beta}\left\|f_{n}\right\|_{L^{2}\left(\mathbb{R}^{n}\right)}^{2}<\infty
$$

we define

$$
\mathcal{G}^{-\beta}=\bigcap_{q \in \mathbb{N}} \mathcal{G}_{-q}^{-\beta}
$$

and equip $\mathcal{G}^{-\beta}$ with the inductive topology. Then $\mathcal{G}^{-\beta}$ is the dual of $\mathcal{G}^{\beta}$, with action

$$
\langle G, F\rangle=\sum_{n=0}^{\infty} n !\left\langle g_{n}, f_{n}\right\rangle .
$$

\section{The Generalized Clark-Ocone Formula}

Now we are prepared to present the main result of this paper. It generalizes the well know Clark-Ocone formula to generalized functions, i.e., to the space $\mathcal{G}^{-\beta}$.

Definition 3.1. Suppose $F=\sum_{n=0}^{\infty} I_{n}\left(f_{n}\right) \in \mathcal{G}^{\beta}$. Then the conditional expectation of $F$ with respect to $\mathcal{F}_{t}$ is given by

$$
E\left[F \mid \mathcal{F}_{t}\right]=\sum_{n=0}^{\infty} \int_{\mathbb{R}^{n}} f_{n} \cdot \chi_{[o, t]^{n}} \mathrm{~d} B^{\otimes n}
$$

Note that this coincides with usual conditional expectation if $F \in L^{2}(\mu)$, and

$$
\left\|E\left[F \mid \mathcal{F}_{t}\right]\right\|_{\mathcal{G}_{k}^{\beta}} \leq\|F\|_{\mathcal{G}_{k}^{\beta}} \text {, for some } k \in \mathbb{N}
$$

In particular

$$
E\left[F \mid \mathcal{F}_{t}\right] \in \mathcal{G}^{-\beta}
$$

Lemma 4.2. Suppose $F, G \in \mathcal{G}^{-\beta}$. Then

$$
E\left[F \diamond G \mid \mathcal{F}_{t}\right]=E\left[F \mid \mathcal{F}_{t}\right] \diamond E\left[G \mid \mathcal{F}_{t}\right]
$$

Proof. Assume that, without loss of generality,

$$
F=\int_{\mathbb{R}^{n}} f_{n} \mathrm{~d} B^{\otimes n}=\sum_{|\alpha|=n} c_{\alpha} \int_{\mathbb{R}^{n}} \xi^{\hat{\otimes} n} \mathrm{~d} B^{\otimes n}
$$

and similarly $G$. By Corollary 2.2 and Definition 4.1, we have

$$
\begin{aligned}
E\left[F \diamond G \mid \mathcal{F}_{t}\right] & =E\left[\int_{\mathbb{R}^{m+n}} f_{n} \hat{\otimes} g_{m} \mathrm{~d} B^{\otimes(m+n)} \mid \mathcal{F}_{t}\right] \\
& =\int_{\mathbb{R}^{m+n}} f_{n} \hat{\otimes} g_{m} \cdot \chi_{[0, t]^{m+n}} \mathrm{~d} B^{\otimes(m+n)} \\
& =\int_{\mathbb{R}^{m+n}} f_{n} \hat{\otimes} \chi_{[0, t]^{n}} \hat{\otimes} g_{m} \cdot \chi_{[0, t]^{m}} \mathrm{~d} B^{\otimes(m+n)} \\
& =E\left[F \mid \mathcal{F}_{t}\right] \diamond E\left[G \mid \mathcal{F}_{t}\right] .
\end{aligned}
$$

\section{Lemma 4.3.}

Let $F \in \mathcal{G}^{-\beta}$. Then $D_{t} F \in \mathcal{G}^{-\beta}$ for a.a. $t \in \mathbb{R}$.

Consider $F, F_{m} \in \mathcal{G}^{-\beta}$ for all $m \in \mathbb{N}$ and

$$
F_{m} \rightarrow F \text { in } \mathcal{G}^{-\beta} .
$$

Then there exists a subsequence $\left\{F_{m_{k}}\right\}_{k=1}^{\infty}$ such that

$$
D_{t} F_{m_{k}} \rightarrow D_{t} F \text { in } \mathcal{G}^{-\beta} \text {, for a.a } t>0
$$


Proof. 1) Suppose $F(\omega)=\sum_{\alpha} c_{\alpha} H_{\alpha}(\omega) \in \mathcal{G}^{-\beta}$. Then

$$
\begin{aligned}
D_{t} F(\omega) & =\sum_{\alpha} c_{\alpha} \sum_{i} \alpha_{i} H_{\alpha-\epsilon^{(i)}}(\omega) \cdot \xi_{i}(t) \\
& =\sum_{\gamma}\left(\sum_{i} c_{\gamma+\epsilon^{(i)}}\left(\gamma_{i}+1\right) \xi_{i}(t)\right) H_{\gamma}(\omega) \\
& =\sum_{\gamma} g_{\gamma}(t) H_{\gamma}(\omega) .
\end{aligned}
$$

where

$$
g_{\gamma}(t)=\sum_{i} c_{\gamma+\epsilon^{(i)}}\left(\gamma_{i}+1\right) \xi_{i}(t) .
$$

Choose $q<\infty$ such that $\|F\|_{\mathcal{G}_{-q}^{-\beta}}^{2}=\sum_{m} \sum_{|\alpha|=m} c_{\alpha}^{2}(\alpha !)^{1-\beta} \mathrm{e}^{-2 q m}<\infty$. We will prove that

$$
\left\|D_{t} F\right\|_{\mathcal{G}_{-q-1}^{-\beta}}^{2}=\sum_{n}\left(\sum_{|\gamma|=n} g_{\gamma}^{2}(\gamma !)^{1-\beta}\right) \mathrm{e}^{-2(q+1) n}<\infty \text { for } \text { a.a.t }
$$

Note that

$$
\int_{\mathbb{R}} g_{\gamma}^{2}(t) \mathrm{d} t=\int_{\mathbb{R}}\left(\sum_{i} c_{\gamma+\epsilon^{(i)}}\left(\gamma_{i}+1\right) \xi_{i}(t)\right)^{2} \mathrm{~d} t=\sum_{\gamma+\epsilon^{(i)}} c^{2}\left(\gamma_{i}+1\right)^{2} .
$$

So

$$
\begin{aligned}
& \sum_{|\gamma|=n}\left(\int_{\mathbb{R}} g_{\gamma}^{2}(t) \mathrm{d} t\right)(\gamma !)^{1+\beta} \\
& =\sum_{\gamma+\epsilon^{(i)}}^{2}\left(\gamma_{i}+1\right)\left(\left(\gamma+\epsilon^{(i)}\right) !\right)^{1+\beta} \\
& \leq \sum_{|\gamma|=n}(n+1) \sum_{i} c_{\gamma+\epsilon^{(i)}}^{2}\left(\gamma_{i}+1\right)\left(\left(\gamma+\epsilon^{(i)}\right) !\right)^{1+\beta} \\
& \leq(n+1) \sum_{|\alpha|=\gamma \mid+1} c_{\alpha}^{2}(\alpha !)^{1+\beta} .
\end{aligned}
$$

Hence, using the fact that $(n+1) \mathrm{e}^{-n} \leq 1$ for all $n$, we get

$$
\begin{aligned}
& \int_{\mathbb{R}}\left\|D_{t} F\right\|_{\mathcal{G}_{-(q+1)}^{-\beta}}^{2} \mathrm{~d} t \\
& =\int_{\mathbb{R}} \sum_{n}\left(\sum_{|\gamma|=n} g_{\gamma}^{2}(\gamma !)^{1-\beta}\right) \mathrm{e}^{-2(q+1) n} \mathrm{~d} t \\
& \leq \sum_{n}(n+1)\left(\sum_{|\alpha|=\gamma \mid+1} c_{\alpha}^{2}(\alpha !)^{1-\beta}\right) \mathrm{e}^{-2(q+1) n} \\
& \leq \sum_{n}\left(\sum_{|\alpha|=|y|+1} c_{\alpha}^{2}(\alpha !)^{1-\beta}\right) \mathrm{e}^{-2 q n} \leq\|F\|_{\mathcal{G}_{-q}^{-\beta}}^{2}<\infty .
\end{aligned}
$$

Therefore,

$$
\left\|D_{t} F\right\|_{\mathcal{G}_{-(q+1)}^{-\beta}}^{2}<\infty \text { for a.a.t }
$$

and

$$
D_{t} F \in \mathcal{G}_{-(q+1)}^{-\beta} \subset \mathcal{G}^{-\beta} \text { for a.a.t }
$$

2) It suffices to prove that if $G_{m} \rightarrow 0$ in $\mathcal{G}_{-q}^{-\beta}$, then there exists a subsequence $\left\{G_{m_{k}}\right\}_{k=1}^{\infty}$ such that $D_{t} G_{m} \rightarrow 0$ in $\mathcal{G}^{-\beta}$ as $k \rightarrow \infty$, for a.a. t. By 
(4.4) we can see that $\left\|D_{t} G_{m}\right\|_{\mathcal{G}_{-q}^{-\beta}} \rightarrow 0$ in $L^{2}(\mathbb{R})$. So there exists a subsequence

$$
\left\{\left\|D_{t} G_{m}\right\|_{\mathcal{G}_{-q}^{-\beta}}\right\}_{k=0}^{\infty}
$$

such that

$$
\left\|D_{t} G_{m}\right\|_{\mathcal{G}_{-q}^{-\beta}} \rightarrow 0 \text { for a.a.t as } k \rightarrow \infty .
$$

Therefore,

$$
D_{t} G_{m_{k}} \rightarrow 0 \text { in } \mathcal{G}^{-\beta} \text { for a.a.t as } k \rightarrow \infty .
$$

The last assertion follows from (4.2).

Theorem 4.4. Suppose $\lambda$ denote Lebesque measure on $\mathbb{R}$. Let $F(\omega) \in L^{2}(\mu)$ be $\mathcal{F}_{t}$-measurable. Then

$$
(t, \omega) \rightarrow E\left[D_{t} F \mid \mathcal{F}_{t}\right](\omega) \in L^{2}(\lambda \times \mu)
$$

and

$$
F(\omega)=E[F]+\int_{0}^{T} E\left[D_{t} F \mid \mathcal{F}_{t}\right] \mathrm{d} B(t) .
$$

Proof. Let $F(\omega)=\sum_{\alpha \in J_{\alpha}} c_{\alpha} H_{\alpha}(\omega)$ be the chaos expansion of $F$ and put

$$
F_{n}=\sum_{\alpha \in J^{\top}} c_{\alpha} H_{\alpha}(\omega)=\sum_{\alpha \in \cdot \sqrt{n}} c_{\alpha} X^{\diamond \alpha},
$$

where $\mathscr{T}_{n}=\{\alpha \in \mathscr{T} ;|\alpha| \leq n \&$ length $(\alpha) \leq n\}$. Then by Lemma 3.8 (see [4]), we have

$$
F_{n}(\omega)=E\left[F_{n}\right]+\int_{0}^{T} E\left[D_{t} F_{n} \mid \mathcal{F}_{t}\right] \mathrm{d} B(t) .
$$

By Itô representation theorem there is a unique $u(t, \omega)$ which is $\mathcal{F}_{t}$ adapted and such that

$$
E\left[\int_{0}^{T} u^{2}(t, \omega) \mathrm{d} t\right]<\infty
$$

and such that

$$
F(\omega)=E[F]+\int_{0}^{T} u(t, \omega) \mathrm{d} B(t),
$$

since $F_{n} \rightarrow F$ in $L^{2}(\mu)$, we conclude that

$$
\begin{aligned}
& E\left[\int_{0}^{T}\left(E\left[D_{t} F_{n} \mid \mathcal{F}_{t}\right]-u(t, \omega)\right)^{2} \mathrm{~d} t\right] \\
& =E\left[F_{n}-F-E\left[F_{n}\right]+E\left[F_{n}\right]^{2}\right] \rightarrow 0 \text { as } n \rightarrow \infty .
\end{aligned}
$$

Therefore,

$$
E\left[D_{t} F_{n} \mid \mathcal{F}_{t}\right] \rightarrow u(t, \omega) \text { in } L^{2}(\lambda \times \mu),
$$

on the other hand, by Lemma 4.1, we have

$$
E\left[D_{t} F_{n} \mid \mathcal{F}_{t}\right] \rightarrow E\left[D_{t} \mid \mathcal{F}_{t}\right] \text { in } \mathcal{G}^{-\beta} \text { for a.a } t
$$

By taking another subsequence, we obtain that

$$
E\left[D_{t} F_{n} \mid \mathcal{F}_{t}\right] \rightarrow u(t, \omega) \text { in } L^{2}(\mu) \text { for a.a } t .
$$


We conclude that

$$
u(t, \omega)=E\left[D_{t} F \mid \mathcal{F}_{t}\right] \text { for a.a } t .
$$

This completes the proof.

Lemma 4.5. Suppose $F \in \mathcal{G}^{-\beta}$ and $f \in \mathcal{G}^{\beta}$. Then

$$
|\langle F, f\rangle| \leq\|F\|_{\mathcal{G}_{-\beta, q}} \cdot\|f\|_{\beta, \hat{q}} ;
$$

where $\hat{q}=\frac{2 q}{\ln 2}$.

Proof. Let $F(\omega)=\sum_{\alpha} a_{\alpha} H_{\alpha}(\omega), f(\omega)=\sum_{\alpha} b_{\alpha} H_{\alpha}(\omega)$. Then

$$
\begin{aligned}
|\langle F, f\rangle| & =\left|\sum_{\alpha} a_{\alpha} b_{\alpha} \alpha !\right|=\left|\sum_{m}\left(\sum_{|\alpha|=m} a_{\alpha} b_{\alpha} \alpha !\right)\right| \\
& \leq\left(\sum_{m}\left(\sum_{|\alpha|=m} a_{\alpha}^{2}\left(\alpha_{i}\right)^{1-\beta}\right) \mathrm{e}^{-2 q m}\right)^{\frac{1}{2}}\left(\sum_{m}\left(\sum_{|\alpha|=m} b_{\alpha}^{2}\left(\alpha_{i}\right)^{1+\beta}\right) \mathrm{e}^{2 q m}\right)^{\frac{1}{2}} \\
& \leq\|F\|_{\mathcal{G}_{-\beta,-q}}\left(\sum_{\alpha} b_{\alpha}^{2}\left(\alpha_{i}\right)^{1+\beta}(2 \mathbb{N})^{\hat{q} \alpha}\right)^{\frac{1}{2}} \\
& =\|F\|_{\mathcal{G}_{-q}^{-\beta}}^{-\beta} \cdot\|f\|_{\hat{q}, \beta} .
\end{aligned}
$$

Lemma 4.6. Suppose $F \in \mathcal{G}^{\beta}, f \in(S)_{\beta}$. Then

$$
\int_{\mathbb{R}}\left\langle E\left[D_{t} F \mid \mathcal{F}_{t}\right], f\right\rangle^{2} \mathrm{~d} t<\infty .
$$

Proof. By Lemma 4.3 and (4.4), we have

$$
\begin{aligned}
& \int_{\mathbb{R}}\left\langle E\left[D_{t} F \mid \mathcal{F}_{t}\right], f\right\rangle^{2} \mathrm{~d} t \leq \int_{\mathbb{R}}\left\|E\left[D_{t} F \mid \mathcal{F}_{t}\right]\right\|_{\mathcal{G}_{-q}^{-\beta}}^{2}\|f\|_{\hat{q}, \beta} \\
& \leq\|f\|_{\hat{q}, \beta} \int_{\mathbb{R}}\left\|\left[D_{t} F \mid \mathcal{F}_{t}\right]\right\|_{\mathcal{G}_{-q}^{-\beta}}^{2}<\infty, \text { for some } q \in \mathbb{N} .
\end{aligned}
$$

Lemma 4.7. Let $F_{n}, F \in \mathcal{G}^{-\beta}$ and $F_{n} \rightarrow F$ in $(S)_{\beta}^{*}$. Then

$$
\int_{0}^{T} E\left[D_{t} F_{n} \mid \mathcal{F}_{t}\right] \diamond W(t) \mathrm{d} t \rightarrow \int_{0}^{T} E\left[D_{t} F \mid \mathcal{F}_{t}\right] \diamond W(t) \mathrm{d} t .
$$

Proof. In case of $\beta=0$ a complete proof is given in [4]. The proof for general $0 \leq \beta<1$ is a simple modification. Note that both integral in (4.6) exist by Lemma 4.7. Hence, by Lemma 4.6 and (4.4), we have

$$
\begin{aligned}
& \left|\left\langle\int_{0}^{T} E\left[D_{t} F_{n} \mid \mathcal{F}_{t}\right] \diamond W(t) \mathrm{d} t-\int_{0}^{T} E\left[D_{t} F \mid \mathcal{F}_{t}\right] \diamond W(t) \mathrm{d} t, f\right\rangle\right| \\
& =\int_{0}^{T}\left|\left\langle E\left[D_{t}\left(F_{n}-F\right) \mid \mathcal{F}_{t}\right], f\right\rangle\right| \mathrm{d} t \\
& \leq \sqrt{T}\|f\|_{\hat{q}, \beta}\left(\int_{0}^{T}\left\|\left[D_{t}\left(F_{n}-F\right) \mid \mathcal{F}_{t}\right]\right\|_{\mathcal{G}_{-q}^{-\beta}}^{2} \mathrm{~d} t\right)^{\frac{1}{2}} \rightarrow 0 \text { as } n \rightarrow \infty .
\end{aligned}
$$

This completes the proof.

Theorem 4.8. Let $F(\omega) \in \mathcal{G}^{-\beta}$ be $\mathcal{F}_{t}$-measurable. Then $E\left[D_{t} F \mid \mathcal{F}_{t}\right] \diamond W(t)$ is integrable in $(S)_{\beta}^{*}$ and

$$
F(\omega)=E[F]+\int_{0}^{T} E\left[D_{t} F \mid \mathcal{F}_{T}\right] \diamond W(t) \mathrm{d} t .
$$


where, $E[F]$ denotes the generalized exsection of $F$.

Proof. Let $F_{n}(\omega)=\sum_{\alpha} c_{\alpha} H_{\alpha}(\omega)$. Then, by Lemma 3.8 (see [4]), we have

$$
F_{n}(\omega)=E\left[F_{n}\right]+\int_{0}^{T} E\left[D_{t} F_{n} \mid \mathcal{F}_{T}\right] \diamond W(t) \mathrm{d} t
$$

therefore,

$$
F(\omega)=E[F]+\lim _{n \rightarrow \infty} \int_{0}^{T} E\left[D_{t} F_{n} \mid \mathcal{F}_{T}\right] \diamond W(t) \mathrm{d} t,
$$

the limit exist in $\mathcal{G}^{-\beta}$ and hence in $(S)_{\beta}^{*}$. The result follows from Lemma 4.7.

\section{References}

[1] Hida, T. (1975) Analysis of Brownian Functionals. Carleton Mathematical Lecture Notes 13, Carleton University, Ottawa.

[2] Oksendal, B., Uboe, J. and Zhang, T.S. (1996) Stochastic Partial Differential Equations-A Modeling, White Noise Functional Approach. Birkhauser, Boston.

[3] Ocone, D. (1994) Malliavin Calculus and Stochastic Integral Representations of Diffusion Processes. Stochastics, 12, 161-185. https://doi.org/10.1080/17442508408833299

[4] Aase, K., Oksendal, B., Privault, N. and Uboe, J. (2000) White Noise Generalizations of the Clark-Haussmann-Ocone Theorem with Application to Mathematical Finance. Finance Stochastic, 4, 465-496. https://doi.org/10.1007/PL00013528

[5] Kachanovsky, N.A. (2011) Clark-Ocone Type Formulas in the Meixner White Noise Analysis. Carpathian Mathematical Publications, 3, 56-72.

[6] Okur, Y.Y. (2012) An Extension of the Clark-Ocone Formula under Benchmark Measure for Lévy Processes. Stochastics-An International Journal of Probability and Stochastic Processes, 84, 251-272. https://doi.org/10.1080/17442508.2010.542817

[7] Hida, T., Kuo, H.H., Potthoff, J. and. Streit, L (1995) White Noise: An Infinite Dimensional Calculus. Kluwer Academic Publishers, Dordrecht.

[8] Kuo, H.H., Potthoff, J. and Streit, L. (1990) A Characterization of White Noise Test Functionals. Nagoya Mathematical Journal, 119, 93-106.

[9] Obata, N. (1994) White Noise Calculus and Fock Space. Lecture Notes in Mathematics, Springer-Verlag, 1577. https://doi.org/10.1007/BFb0073952

[10] Kondratiev, Y.G. and Streit, L. (1993) Spaces of White Noise Distributions Constructions, Descriptions, Applications I. BiBoS Preprint No. 510. Reports on Mathematical Physics, 33, 341-366. https://doi.org/10.1016/0034-4877(93)90003-W

[11] Kondratiev, Y.G., Leukert, P. and Streit, L. (1996) Wick Calculus in GAUSSIAN analysis. Acta Applicandae Mathematicae, 44, 269-294.

[12] Kuo, H.H. (1997) Stochastic Integration via White Noise Analysis. Nonlinear Analysis: Theory, Methods \& Applications, 30, 317-328. https://doi.org/10.1016/S0362-546X(96)00221-0

[13] Kuo, H.H. (1996) White Noise Distribution Theory. CRC Press, Boca Raton.

[14] Potthoff, J. and Timpel, M. (1995) On a Dual Pair of Spaces of Smooth and Generalized Random Variables. Potential Analysis, 4, 637-654. https://doi.org/10.1007/BF02345829

[15] Ito, K. (1951) Multiple Wiener Integral. Journal of the Mathematical Society of Japan, 3, 157-169. https://doi.org/10.2969/jmsj/00310157 